\title{
Surveying and fast detection of Ralstonia solanacearum bacterium in some Egyptian governorates
}

\author{
Hanafy, M.S. *, G.M. El-Habbaa**, F.G. Mohamed**, N. M. Balabel* and G.A. Ahmed** \\ * Bacterial Diseases Research Dept., Plant Pathology Research Institute, Agric. Res. Center (ARC), Giza, Egypt \\ ***Plant Pathol. Dept., Fac. Agric., Moshtohor, Benha Univ., Egypt \\ Correspondence author: ghabaa@yahoo.com \\ Mohamed.soliman304@hotmail.com
}

\begin{abstract}
Naturally infected potato plants showing bacterial wilt symptoms were collected from different habitats and used for isolation of $R$. solanacearum pathogen. Also, isolation was carried out from soil, water canals, weeds and some other common crops (Pepper, Tomato, Banana, Snap Bean, Corn, Eggplant, Onion, Cabbage and Clover). All isolates showed typical and atypical morphological growth of $R$. solanacearum on Selective Medium South Africa medium (SMSA). Out of suspected 321 isolates of $R$. solanacearum, only 209 isolates were positive when identified using Immunofluorescence antibody stain test (IFAS) while the rest were negative. When these 209 isolates were identified again by growing on SMSA medium, only 194 isolates were positive as typical forms of $R$. solanacearum while the rest15 isolates appeared as atypical forms of $R$. solanacearum. Selected sixty-six isolates of those identified as typical or atypical forms of $R$. solanacearum were tested for their virulence. Results indicate that all tested 66 isolates could infect potato plants (cv. Spunta), where disease severity (DS \%) ranged between 26.4\% - $100 \%$ when incubated for 20 days. In this respect, MktT-17 (Menofia - EL Kawady - typical tuber isolate) isolate was the highest infective one. While, the least infective isolate was BhhaWa-53 (Beheira Kom Hamada - atypical - water) isolate, at the same period of incubation. Also, the rest of other isolates were infective and caused bacterial wilt symptoms below $98.4 \%$ and over $28.0 \%$. All atypical forms of $R$. solanacearum isolates were less infective than the typical forms which were more virulent in this respect. Also, all sixty-six tested isolates of typical or atypical $R$. solanacearum were infective and caused bacterial wilt symptoms of tomato plants (cv. Ponto) which reached $100 \%$ DS but they differed in the time of incubation. The typical isolates were more virulent and fast in their infection on tomato plants (cv. Ponto). The highest DS\% on tomato plants (cv. Ponto) was recorded with the typical $R$. solanacearum isolate MktT-17 (Menofia - EL Kawady - typical - tuber) to be $100 \%$ at 4 days of incubation period. While, the lowest DS\% was recorded with MmtOB-47 (Menofia - Om saber - typical - other common crop - Banana) isolate where it recorded 100\% DS at 10 days of incubation period. Moreover, the fastest atypical form of $R$. solanacearum was GhzaS-55 (Gharbia - Kafr el Zayat - atypical - Soil) isolate which causing $100 \%$ wilt DS on tomato plants (cv. Ponto) at 9 days of incubation period while, the slowest atypical ones were BhhaS-56 (Beheira - Kom Hamada - atypical - soil), GdaS-62 (Giza - Wardan - atypical soil) and GHzaOT-64 (Gharbia- Kafr el zayat atypical- other common crop - Tomato) isolate which caused $100 \%$ wilt DS on tomato plants (cv. Ponto) at 15 days of incubation period.
\end{abstract}

Key words: Wilt and brown rot diseases, typical and atypical forms, Potato plants, $R$. solanacearum, Virulence.

\section{Introduction}

Potato (Solanum tuberosum L.) is considered one of the major 10 important food crops around the world with total production of 376 million tons. In Egypt, it represents the second exporting crop after oranges where, about 428 thousand tons were exported to many countries especially the EU countries and Russia (FAO Stat 2013). In addition, it ranked as the sixth crop in total production after sugarcane, sugar beet, maize, wheat and rice with total production of 503 thousand tons (FAO Stat 2016). Bacterial plant diseases are the most critical factors limiting production of the agricultural sector in most countries and often difficult to control. One of the most important bacterial diseases of potatoes is brown rot disease which caused by Ralstonia solanacearum where it predominates in tropical, subtropical and temperate regions and this disease is one of the major constraints to production of solanaceous crops in these regions (Fahy and Persley 1983, Hayward 1991). In 1896 the causal agent of bacterial wilt was first identified and described as Bacillus solanacearum by Erwin F. Smith. In 1914, Smith changed the name to Pseudomonas solanacearum and for almost 80 years, the pathogen was grouped within the genus Pseudomonas. In 1992, the new genus Burkholderia was validated and reclassified $P$. solanacearum to become Burkholderia solanacearum which changed to Ralstonia solanacearum in 1995 (Smith, 1896 \& 1914 and Yabuüchi et al. 1992 \& 1995). $R$. solanacearum is the causative agent of potato bacterial wilt. This pathogen is a heterogeneous and soil-borne plant pathogen, five races have been described revering to the hosts affected and five biovars revering to their ability to utilize and /or oxidize several hexose alcohols and disaccharides (Hayward, 1991). In Egypt, the dominant race of $R$. 
solanacearum is race 3 , biovar 2 which is widely spreading in Europe, suggesting a possible origin of introducing to Egypt with seed tubers (Farag, 2000). Currently, the species complex $R$. solanacearum is divided into four phylotypes, characterized by high genetic distances and genomic rearrangements, based on the geographical location; phylotype I (Asia), phylotype IIA and IIB (America), phylotype III (Africa) and phylotype IV (Indonesia). (Fegan \& Prior, 2005).

This work aimed to study the occurrence of potato brown rot disease in different Egyptian governorates and throw the light on the different hosts of the causal of brown rot pathogen.

\section{Materials \& Methods}

\section{Isolation of Ralstonia solanacearum from different habitats:}

Out of 221 samples were collected from different habitats (tubers, soil, water canals and weeds) through the following governorates Menofia, Gharbia, Beheira, Beni Suef and Giza.

\subsection{Isolation from potato tubers:}

Naturally infected samples of potato plants showing external and internal symptoms of potato brown rot disease were collected from potato cultivation sites in different governorates. These samples were investigated in the Potato Brown Rot Project (PBRP), Dokki, Giza, Egypt. In this respect, the infected potato tubers were washed in running tap water, surface sterilized with $90 \%$ ethyl alcohol by flaming then the stolon ends were aseptically removed using sharp sterilized knife. Cores of 5-10 mm diameter and $5 \mathrm{~mm}$ length, containing mainly vascular and cortical tissues were macerated in $1.0 \mathrm{~mL}$ of sterile phosphate buffer; then the suspension was transferred to sterile $1 \mathrm{~mL}$ Eppendorf tubes. The suspension was left to stand for $30 \mathrm{~min}$. Plating was made on modified Selective Medium South Africa (SMSA) which consists of basal medium + Crystal violet $0.5 \mathrm{~mL}$, Polymixin-B-sulphate $10 \mathrm{~mL}$, Bacitracin 7.5 mL, Chloromphenicol $2.5 \mathrm{~mL}$, Penicillin-G $1 \mathrm{~mL}$ and 2,3,5 triphenyltetrazolium chloride salts $5 \mathrm{~mL}$. This medium is considered as semi selective medium for isolation of $R$. solanacearum as described by Engelbrecht, (1994) and modified by Elphinstone, (1996).

Purification was made by streaking and plating for typical and atypical colonies on basal medium which consists of: $1.0 \mathrm{~g}$ casamino acid, $10.0 \mathrm{~g}$ bactopeptone, $5.0 \mathrm{~mL}$ glycerol, $20.0 \mathrm{~g}$ agar and $1000.0 \mathrm{~mL}$ distilled water (pH 6.9). And on King's medium (20.0 g Proteose peptone, $1.5 \mathrm{~g} \mathrm{~K}_{2} \mathrm{HPO}_{4}, 1.5 \mathrm{~g} \mathrm{MgSO}_{4}, 15 \mathrm{~mL}$ glycerol, $20.0 \mathrm{~g}$ agar and $1000 \mathrm{~mL}$ distilled water, $\mathrm{pH}$ 7.2) then incubated at $28^{\circ} \mathrm{C}$ and daily observed (approx. 3 days) for developing less fluidal, red to dark red center and opaque colonies which considered as atypical colony forms for Ralstonia solanacearum. While fluidal, slightly raised, irregular white or white with pink center colonies considered as typical colony forms of Ralstonia solanacearum. Colonies were selected, picked up and streaked on glucose nutrient agar medium, incubated for $48 \mathrm{hr}$ at $28^{\circ} \mathrm{C}$ (Dowson, 1957) for further studies.

\subsection{Isolation from soil:}

Soil samples were collected from the same mentioned above governorates to isolate the pathogenic bacteria. About $200 \mathrm{~g}$ soil was taken from $30 \mathrm{~cm}$ depth using a sampling auger from each sampling spot in each field and then mixed together for homogenization. In the laboratory, 10 grams of soil samples were suspended in $250 \mathrm{~mL}$ flask contains $90 \mathrm{~mL}$ of sterilized phosphate buffer $(0.01 \mathrm{M})$ and were shaken at $100 \mathrm{rpm}$ at $15^{\circ} \mathrm{C}$ for two hrs. Then isolation was carried on SMSA medium by spreading $100 \mu \mathrm{L}$ per sample and incubated at $28^{\circ} \mathrm{C}$ for 3 days. (Wenneker et al., 1999).

\subsection{Isolation from irrigation water:}

Water sampling points on streams were chosen near to potato fields and other inspected fields (up and down stream of the potato fields and other inspected fields) $50 \mathrm{~mL} / \mathrm{sample}$ were collected at a depth of 30$40 \mathrm{~cm}$ in $50 \mathrm{~mL}$ disposable centrifuge tubes then placed in an icebox. In the laboratory, the tubes were centrifuged at $10.000 \mathrm{rpm}$ for 15 minutes at $15^{\circ} \mathrm{C}$ by using Sigma $3-18 \mathrm{~K}$ centrifuge. The sediment was resuspended in $1 \mathrm{~mL}$ sterile phosphate buffer $(0.01 \mathrm{M})$ and vortexed for homogenization. Plating was made on modified SMSA medium and incubated at $28^{\circ} \mathrm{C}$ for 3-5 days. (Janse, 1988 \& 1996; Janse \& Schans, 1998 and Wenneker et al., 1999).

\subsection{Isolation from weeds:}

Different weed plants were collected from the inspected fields of the governorates mentioned above. Weeds were defined (Zaki, 1991 \& 2000) as Rumex dentatus (toothed dock), Solanum nigrum (Black nightshade), Portulaca oleracea (little hogweed) and Malva parviflora (Cheese weed). Roots plus the crown parts were thoroughly washed under tap water and disinfected in $70 \%$ ethyl alcohol. These parts were macerated in $1 \mathrm{~mL}$ sterile phosphate buffer $0.1 \mathrm{M}$ and left for 10 min then $100 \mu \mathrm{L}$ per sample were spread onto SMSA medium and incubated at $28^{\circ} \mathrm{C}$ for 3 days as described by Pradhanang et al., (2000).

\section{Identification of isolated bacteria:}

Different methods were used to identify and study differences between isolates as following:

\subsection{Cultural characteristics on SMSA medium:}

Isolated bacteria were re-inoculated on SMSA medium to determine the typical and atypical forms of isolated $R$. solanacearum isolates as mentioned by Engelbrecht (1994) and Elphinstone et al. (1996). 2.2. Immunofluorescence antibody stain (IFAS):

Suspensions of the selected $R$. solanacearum isolates were prepared from grown cultures for $48 \mathrm{~h}$ and adjusted to a standard optical density at $590 \mathrm{~nm}$ to $10^{6}$ colonies forming units $(\mathrm{cfu} / \mathrm{mL})$ using Jenway 
6300 spectrophotometer, then a standard volume of 20 $\mu \mathrm{L}$ of each suspension was pipetted onto multi-well slides $6 \mathrm{~mm}$ window diameter. Slides were left to dry on a Stuart CB500 hot plate at $40^{\circ} \mathrm{C}$ and gently heatfixed by flaming (PBRP protocol, Egypt). All windows were covered with $25 \mu \mathrm{L}$ of the antiserum (anti $R$. solanacearum polyclonal) in four dilutions $(1: 800,1: 1600,1: 3200$ and 1:6400) and incubated in a humid tray chamber for $30 \mathrm{~min}$ at room temperature then washed with tween buffer and $0.01 \mathrm{M}$ phosphate buffer (PB). Add $25 \mu \mathrm{L}$ of anti-rabbit Nordic SW/AR fluorescein isothiocyanate conjugate in a 100-fold dilution (prepared in phosphate buffer saline) to each window and incubate for $30 \mathrm{~min}$ in a humid tray chamber. Slides then washed with tween buffer and $0.01 \mathrm{M} \mathrm{PB}$, and the excess moisture was removed carefully by blotting with filter or tissue paper. One droplet of $0.1 \mathrm{~mol}^{-1}$ phosphate glycerin buffer ( $\mathrm{pH}$ 7.6) was added to each window and the slides were covered with long cover glasses. Slides were examined with Olympus bx51 microscope (tube factor 1.25 ) with an epifluerescent light source and suitable filters with FITC, using a $100 \mathrm{X}$ oil immersion objective and a $10 \mathrm{X}$ eyepiece. At least 20 microscope fields per window were scanned for the presence of morphologically typical fluorescing cells (Janse, 1988).

\section{Virulence of $\boldsymbol{R}$. solanacearum isolates:}

Sixty-six isolates were selected based on their occurrence in each governorate, location, form of each isolate and source of isolates (water, tuber, weed, soil and/or other common crops in the same region). So, all governorates and locations were represented in addition to all other common crop soil isolates. Firstly, pure cultures of the selected 66 bacterial isolates were primary identified and confirmed as Ralestonia solanacearum using IFAS and SMSA tests. These isolates were grown on casmino acid peptone glucose (CPG) agar medium (5.0 g Dextrose, $10.0 \mathrm{~g}$ Peptone, $1.0 \mathrm{~g}$ Casamino acid, $20.0 \mathrm{~g}$ Agar and $1000 \mathrm{~mL}$ Distilled water, $\mathrm{pH} 7.2$ ) at $28^{\circ} \mathrm{C}$ for $48 \mathrm{~h}$ and suspended in sterile phosphate buffer $0.05 \mathrm{M} \mathrm{pH} \mathrm{7.2.} \mathrm{The}$ prepared suspensions were adjusted to a standard optical density $590 \mathrm{~nm}$ to obtain $10^{7} \mathrm{cfu} / \mathrm{mL}$ (Kelman, 1954). Potato tubers (cv. Spunta) and tomato plants (cv. Ponto) were examined to be free from $R$. solanacearum using IFAS and planting on SMSA media before using in this experiment. Four weeks old, tomato seedlings were transplanted in $10 \mathrm{~cm}$ diameter pots, containing sterilized sand-clay soil mixture $(1: 1 \mathrm{v} / \mathrm{v})$ while, potato tubers which previously stored at $4{ }^{\circ} \mathrm{C}$ were placed in trays at room temperature in dark to stimulate germination. Germinated tubers were planted in pots $(30 \mathrm{~cm}$ diameter) containing $5 \mathrm{Kg}$ sterilized sand-clay soil (1:1 $\mathrm{v}: \mathrm{v})$, where one germinated tuber was planted in each pot. Tomato plants (three-weeks after transplanting) were injected at the leaf axis with a sterile fine needle laden with the bacterial growth of the pathogen
(Janse, 1988). Potato plants were inoculated into the axel of the second and third leaf from the apical meristem by injection of the bacterial suspension $(10 \mu \mathrm{L})$ using a sterile fine needle (Martin and ElNashaar, 1992). The inoculated plants were covered with polyethylene bags for three days at $30^{\circ} \mathrm{C}$ and $28 \%$ relative humidity $(\mathrm{RH})$ in an automated quarantine greenhouse, then bags were removed and pots were irrigated daily.

Wilt symptoms severity were recorded daily using the scale of Kempe and Sequeira (1983) where, $(0=$ no symptoms, $1=$ up to $25 \%$ wilt, $2=26-50 \%$ wilt, 3 $=51-75 \%$ wilt, $4=76-100 \%$ wilt and $5=$ dead plants).

\section{Results \& Discussion}

\section{Isolation of Ralstonia solanacearum:}

Naturally infected potato plants from different habitats showing brown rot diseases were used for isolation of $R$. solanacearum pathogen. Also, isolation was carried out from soil, water canals and some other common crops which cultivated commonly in the region of potato cultivations (i.e., Pepper $=\mathrm{P}$, Tomato $=\mathrm{T}$, Banana $=\mathrm{B}$, Snap Bean $=\mathrm{Sb}$, Corn $=\mathrm{C}$, Eggplant $=\mathrm{E}$, Onion=O, Cabbage $=\mathrm{Ca}$ and $\mathrm{Clover}=\mathrm{Cl}$ ) as clear in Table 1. All isolates showed typical and atypical morphological growth of $R$. solanacearum on SMSA medium. Where from total of 321 suspected isolates, 21 isolates were isolated from irrigation water, 160 isolates were isolated from potato tubers, 36 isolates of potato cultivation soil and 61 isolates of weed plants which grown in potato fields. Also, 8 suspected isolates were isolated from common field crop plants and 35 isolates were isolated from soil of the cultivated common field crops. The colonies for typical forms were fluidal white with red center while, for atypical forms less fluidal, red to dark red center and opaque colonies. These results could be discussed in light the findings of Abd El-Ghany (2010) who surveyed the causal of brown rot disease infecting potato cultivations in many localities of four Egyptian governorates. This survey was done on potato tubers, water, soil and some grown weed plants in potato fields and borders of irrigation canals. The highest percentage of positive samples were in Menofia followed by Gharbia and Kalubia while the lowest one was in Ismailia governorate. Also, Hagag (2015) used SMSA medium for isolation of $R$. solanacearum from soil, tuber and weed samples of different Egyptian governorates. Moreover, Ali (2017) isolated the pathogen on SMSA medium from different samples collected from infected potato tubers, field soils with previous disease history, irrigation water and weeds from Ismailia and Menofia governorates. These isolates show bacteriological characteristic like those described for race 3 biovar 2 Ralstonia solanacearum as described in Bergey's Manual of Systematic Bacteriology. 
Table 1. General screening of suspected $R$. solanacearum isolates on SMSA medium from different habitats which collected from different governorates:

\begin{tabular}{|c|c|c|c|c|c|c|c|}
\hline \multirow{3}{*}{ Governorate } & \multirow{3}{*}{ Location } & \multicolumn{6}{|c|}{ Number of suspected Ralstonia solanacearum isolates } \\
\hline & & \multicolumn{4}{|c|}{ Potato Fields } & \multicolumn{2}{|c|}{ Common Crops } \\
\hline & & $\mathbf{W a}$ & $\mathbf{T}$ & $\mathbf{S}$ & We & $\mathbf{P l}$ & $\mathbf{S}$ \\
\hline \multirow{5}{*}{$\begin{array}{c}\text { Gharbia } \\
\text { (Gh) }\end{array}$} & & & & & & $2 \mathrm{~T}$ & $3 \mathrm{~T}$ \\
\hline & Kafr EL zayat (z) & 3 & 44 & 14 & 15 & $\mathrm{OE}$ & $6 \mathrm{E}$ \\
\hline & & & & & & 10 & $2 \mathrm{O}$ \\
\hline & Gafarya (g) & 2 & 24 & 2 & 14 & $0 \mathrm{C}$ & $1 \mathrm{C}$ \\
\hline & & & & & & $\mathrm{OCl}$ & $2 \mathrm{Cl}$ \\
\hline Beheira (Bh) & Kom Hamada (h) & 9 & 7 & 6 & 7 & $1 \mathrm{~T}$ & $2 \mathrm{~T}$ \\
\hline \multirow{3}{*}{ Menofia (M) } & Talia (i) & 2 & 10 & 2 & 5 & $\begin{array}{c}\mathrm{Ca} \\
\mathrm{OSb}\end{array}$ & $\begin{array}{c}1 \mathrm{Ca} \\
1 \mathrm{Sb}\end{array}$ \\
\hline & Om Saber (m) & 2 & 20 & 4 & 7 & $\begin{array}{c}0 \mathrm{~B} \\
0 \mathrm{Sb} \\
0 \mathrm{C} \\
0 \mathrm{Cl} \\
\end{array}$ & $\begin{array}{c}2 \mathrm{~B} \\
1 \mathrm{Sb} \\
1 \mathrm{C} \\
2 \mathrm{Cl} \\
\end{array}$ \\
\hline & $\begin{array}{c}\text { El Kawady } \\
\text { (k) }\end{array}$ & 3 & 10 & 3 & 6 & $1 \mathrm{P}$ & $1 \mathrm{P}$ \\
\hline Beni Suef & Kafr Mymon (y) & 0 & 9 & 2 & 4 & $0 \mathrm{O}$ & $2 \mathrm{O}$ \\
\hline (Bs) & & & & 2 & & $1 \mathrm{~T}$ & $1 \mathrm{~T}$ \\
\hline Giza & Wardan & 0 & 36 & 3 & 3 & $1 \mathrm{P}$ & $3 \mathrm{P}$ \\
\hline$(\mathbf{G})$ & (d) & & & & & $1 \mathrm{~T}$ & $4 \mathrm{~T}$ \\
\hline Sub-Total & & 21 & 160 & 36 & 61 & 8 & 35 \\
\hline Total & & & & & & & \\
\hline
\end{tabular}

Where: Common crops= crops commonly cultivated in the region of potato either pre or post cultivation.

$\mathrm{Wa}=$ Water, $\mathrm{T}=$ Tuber, $\mathrm{S}=$ Soil, $\mathrm{We}=$ Weeds, $\mathrm{Pl}=\mathrm{Plant} . \mathrm{P}=$ Pepper, $\mathrm{T}=$ Tomato, $\mathrm{B}=\mathrm{B}$ anana, $\mathrm{Sb}=\mathrm{Snap} \mathrm{Beans}$, $\mathrm{C}=$ Corn, $\mathrm{E}=$ Eggplant, $\mathrm{O}=$ Onion, $\mathrm{Ca}=\mathrm{Cabbage}$ and $\mathrm{Cl}=$ Clover

\section{Preliminary identification of suspected $R$. solanacearum isolates using IFAS test and planting on SMSA media:}

Preliminary identification was done of suspected isolates from different habitats which isolated on the selective SMSA medium (total 321 isolates) using IFAS test as clear in Table 2. In this respect, results show that all 21 bacterial isolates of water were positive as $R$. solanacearum using IFAS test while, among 160 bacterial isolates of tubers only 147 were positive as $R$. solanacearum and 13 isolates were negative. On the other hand, among 36 bacterial isolates of soil, only 24 isolates were positive and 12 isolates were negative. Also, among 61 bacterial isolates of weeds, only 7 isolates were positive as $R$. solanacearum. As for the bacterial isolates of other common field crop plants (Table 3), all 8 isolates were negative while, among 35 isolates of soil of cultivated common field crop, only 10 isolates were positive as $R$. solanacearum using IFAS test. As for the identification of the suspected isolates of $R$. solanacearum (321 isolates) using SMSA medium test, Results in Tables 4 indicate that among 21 bacterial isolates of water, only 18 isolates were typical form of $R$. solanacearum while the rest 3 isolates were atypical form of $R$. solanacearum. Also, among 160 isolates of potato tubers, only 147 isolates were typical form of $R$. solanacearum while the rest 13 isolates were negative as $R$. solanacearum when confirmed by IFAS test. As for soil isolates, among 36 isolates, only 16 isolates were typical form of $R$. solanacearum while the rest 8 isolates were atypical form isolates in addition to 12 isolates were negative as $R$. solanacearum when confirmed for identification using IFAS test. Also, among 61 isolates of weeds in potato fields, only 6 isolates were typical form of $R$. solanacearum and only one was as atypical form isolate of $R$. solanacearum whereas the rest 54 isolates were negative as $R$. solanacearum when confirmed for identification using IFAS test. Regarding the suspected isolated bacteria from other common field crop plants (Table 5), all 8 isolates were negative as $R$. solanacearum when they confirmed for their identification again using IFAS test where they were not typical or atypical forms on SMSA medium test. Meanwhile, among 35 isolates of soil of cultivated common field crop, 7 isolates were typical form as $R$. solanacearum and 3 isolate were atypical forms of $R$. solanacearum while the rest 25 isolates were negative as $R$. solanacearum when confirmed again for identification using IFAS test. Also, it is clear from data in Table (6) that out of suspected 321 isolates of $R$. solanacearum, only 209 isolates were positive when identified using IFAS test while the rest were negative. When these 209 isolates were identified again by growing on SMSA medium, only 194 isolates were positive as typical forms of $R$. solanacearum while the rest 15 isolates appeared as atypical forms of $R$. solanacearum. For further studies, sixty-six isolates of those identified as typical 
or atypical forms of $R$. solanacearum were selected and grouped as clear in Table 7 based on governorate and location of isolation, habitats of isolation (water, tubers, soil, weeds) and other crops of isolation (pepper, tomato, eggplant and banana). These isolates were coded as clear in Table $\mathbf{8}$. These results could be interpreting in light the findings of Atta (2008) who stated that detection methods by plating on the SMSA medium resulting colonies with irregularly round shape and slimy white color with pink centers, which considered the typical morphology of bacterial colony. Also, Ali (2017) stated that culturing on
SMSA medium is the best method to detect the pathogen. IFAS is of limited interest in detection in soil because the assayed soil extracts caused a strong background fluorescence that hampered the visualization of target cells by IFAS. Meanwhile, Mikhail et al. (2017) observed two different types of colony the first, was the virulent type or typical which was highly fluidal, irregular, white or cream with pink centers. While the second, was the avirulent type or atypical was uniformly round, smaller and completely pink to deep red colonies.

Table 2. Preliminary identification of Ralstonia solanacearum isolates which isolated from different habitats of potato fields in some Egyptian governorates using IFAS test

No. of positive and negative isolates from different habitats

\begin{tabular}{|c|c|c|c|c|c|c|c|c|c|c|c|c|c|}
\hline \multirow[t]{2}{*}{ Governorate } & \multirow[t]{2}{*}{ Location } & \multicolumn{3}{|c|}{ Water } & \multicolumn{3}{|c|}{ Tubers } & \multicolumn{3}{|c|}{ Soil } & \multicolumn{3}{|c|}{ Weeds } \\
\hline & & TSI & + & - & TSI & + & - & TSI & + & - & TSI & + & - \\
\hline \multirow{2}{*}{$\begin{array}{c}\text { Gharbia } \\
\text { (Gh) }\end{array}$} & $\begin{array}{l}\text { Kafr EL } \\
\text { zayat (z) }\end{array}$ & 3 & 3 & 0 & 44 & 41 & 3 & 14 & 3 & 11 & 15 & 1 & 14 \\
\hline & $\begin{array}{c}\text { Gafarya } \\
(\mathrm{g})\end{array}$ & 2 & 2 & 0 & 24 & 22 & 2 & 2 & 1 & 1 & 14 & 2 & 12 \\
\hline Beheira (Bh) & $\begin{array}{c}\text { Kom } \\
\text { Hamada } \\
(\mathrm{h}) \\
\end{array}$ & 9 & 9 & 0 & 7 & 6 & 1 & 6 & 6 & 0 & 7 & 0 & 7 \\
\hline \multirow{3}{*}{ Menofia (M) } & Talia (i) & 2 & 2 & 0 & 10 & 10 & 0 & 2 & 2 & 0 & 5 & 1 & 4 \\
\hline & $\begin{array}{c}\text { Om Saber } \\
(\mathrm{m})\end{array}$ & 2 & 2 & 0 & 20 & 18 & 2 & 4 & 4 & 0 & 7 & 1 & 6 \\
\hline & $\begin{array}{c}\text { El Kawady } \\
(\mathrm{k})\end{array}$ & 3 & 3 & 0 & 10 & 10 & 0 & 3 & 3 & 0 & 6 & 1 & 5 \\
\hline $\begin{array}{l}\text { Beni Suef } \\
\quad(\text { Bs })\end{array}$ & $\begin{array}{c}\text { Kafr } \\
\text { Mymon } \\
(\mathrm{y}) \\
\end{array}$ & 0 & 0 & 0 & 9 & 9 & 0 & 2 & 2 & 0 & 4 & 0 & 4 \\
\hline Giza $(G)$ & $\begin{array}{c}\text { Wardan } \\
\text { (d) }\end{array}$ & 0 & 0 & 0 & 36 & 31 & 5 & 3 & 3 & 0 & 3 & 1 & 2 \\
\hline \multicolumn{2}{|c|}{ Total } & 21 & 21 & $\mathbf{0}$ & 160 & 147 & 13 & 36 & 24 & 12 & 61 & 7 & 54 \\
\hline
\end{tabular}

TSI $=$ Total suspected isolates, $(+)=$ Positive IFAS, $(-)=$ Negative IFAS

Table 3. Preliminary identification of Ralstonia solanacearum isolates of the cultivated common field crops in some Egyptian governorates using IFAS test.

\begin{tabular}{|c|c|c|c|c|c|c|c|}
\hline \multirow{3}{*}{ Governorate } & \multirow{3}{*}{ Location } & \multicolumn{6}{|c|}{$\begin{array}{l}\text { No. of positive and negative Isolates from other common } \\
\text { field crops }\end{array}$} \\
\hline & & \multicolumn{3}{|c|}{ Plant } & \multicolumn{3}{|c|}{ Soil } \\
\hline & & TSI & + & - & TSI & + & - \\
\hline \multirow[t]{2}{*}{ Gharbia (Gh) } & Kafr EL zayat (z) & $\begin{array}{l}2 \mathrm{~T} \\
0 \mathrm{E} \\
1 \mathrm{O} \\
\end{array}$ & 0 & 3 & $\begin{array}{l}3 \mathrm{~T} \\
6 \mathrm{E} \\
2 \mathrm{O} \\
\end{array}$ & $\begin{array}{l}1 \mathrm{~T} \\
2 \mathrm{E}\end{array}$ & 8 \\
\hline & Gafarya (g) & $\begin{array}{c}0 \mathrm{C} \\
0 \mathrm{Cl} \\
\end{array}$ & 0 & 0 & $\begin{array}{c}1 \mathrm{C} \\
2 \mathrm{Cl} \\
\end{array}$ & 0 & 3 \\
\hline Beheira (Bh) & Kom Hamada (h) & $1 \mathrm{~T}$ & 0 & 1 & $2 \mathrm{~T}$ & $1 \mathrm{~T}$ & 1 \\
\hline \multirow{3}{*}{ Menofia (M) } & Talia (i) & $\begin{array}{c}0 \mathrm{Ca} \\
0 \mathrm{Sb} \\
\end{array}$ & 0 & 0 & $\begin{array}{l}\mathrm{Ca} \\
1 \mathrm{Sb}\end{array}$ & 0 & 2 \\
\hline & $\begin{array}{c}\text { Om Saber } \\
\text { (m) }\end{array}$ & $\begin{array}{c}\text { OB } 0 \mathrm{Sb} \\
0 \mathrm{C} \\
0 \mathrm{Cl}\end{array}$ & 0 & 0 & $\begin{array}{c}2 \mathrm{~B} \\
1 \mathrm{Sb} \\
1 \mathrm{C} \\
2 \mathrm{Cl}\end{array}$ & $\begin{array}{r}1 \mathrm{~B} \\
1 \mathrm{Sb}\end{array}$ & 4 \\
\hline & $\begin{array}{l}\text { El Kawady } \\
(\mathrm{k})\end{array}$ & $1 \mathrm{P}$ & 0 & 1 & $1 \mathrm{P}$ & $1 \mathrm{P}$ & 0 \\
\hline
\end{tabular}




\begin{tabular}{|c|c|c|c|c|c|c|c|}
\hline $\begin{array}{c}\text { Beni Suef } \\
\text { (Bs) }\end{array}$ & Kafr Mymon (y) & $\begin{array}{l}0 \mathrm{O} \\
1 \mathrm{~T} \\
\end{array}$ & 0 & 1 & $\begin{array}{l}2 \mathrm{O} \\
1 \mathrm{~T} \\
\end{array}$ & $1 \mathrm{~T}$ & 2 \\
\hline Giza $(\mathbf{G})$ & Wardan (d) & $\begin{array}{l}\mathrm{P} \\
1 \mathrm{~T}\end{array}$ & 0 & 2 & $\begin{array}{l}3 \mathrm{P} \\
4 \mathrm{~T}\end{array}$ & $\begin{array}{l}1 \mathrm{P} \\
1 \mathrm{~T}\end{array}$ & 5 \\
\hline & & 8 & $\mathbf{0}$ & 8 & 35 & 10 & 25 \\
\hline
\end{tabular}

Where: $\mathrm{P}=$ Pepper, $\mathrm{T}=$ Tomato, $\mathrm{B}=\mathrm{B}$ anana, $\mathrm{Sb}=$ Snap Beans, $\mathrm{E}=$ Eggplant

$(+)=$ Positive IFAS,$(-)=$ Negative IFAS.

Table 4. Preliminary identification of Ralstonia solanacearum isolates which isolated from different habitats of potato fields in some Egyptian governorates using SMSA medium test

\begin{tabular}{|c|c|c|c|c|c|c|c|c|c|c|c|c|c|}
\hline \multirow{3}{*}{ Governorate } & \multirow{3}{*}{ Location } & \multicolumn{12}{|c|}{ No. of typical and atypical isolates from different habitats } \\
\hline & & \multicolumn{3}{|c|}{ Water } & \multicolumn{3}{|c|}{ Tubers } & \multicolumn{3}{|c|}{ Soil } & \multicolumn{3}{|c|}{ Weeds } \\
\hline & & TSI & $\mathbf{t}$ & $\mathbf{a}$ & TSI & $\mathbf{t}$ & $\mathbf{a}$ & TSI & $\mathbf{t}$ & $\mathbf{a}$ & TSI & $\mathbf{t}$ & $\mathbf{a}$ \\
\hline \multirow{2}{*}{$\begin{array}{c}\text { Gharbia } \\
\text { (Gh) }\end{array}$} & $\begin{array}{c}\text { Kafr EL zayat } \\
(\mathrm{z})\end{array}$ & 3 & 2 & 1 & 44 & 41 & 0 & 14 & 2 & 1 & 15 & 1 & 0 \\
\hline & Gafarya $(\mathrm{g})$ & 2 & 2 & 0 & 24 & 22 & 0 & 2 & 1 & 0 & 14 & 2 & 0 \\
\hline Beheira (Bh) & $\begin{array}{c}\text { Kom Hamada } \\
\text { (h) }\end{array}$ & 9 & 8 & 1 & 7 & 6 & 0 & 6 & 4 & 2 & 7 & 0 & 0 \\
\hline \multirow{3}{*}{ Menofia (M) } & Talia (i) & 2 & 2 & 0 & 10 & 10 & 0 & 2 & 2 & 0 & 5 & 1 & \\
\hline & Om Saber (m) & 2 & 2 & 0 & 20 & 18 & 0 & 4 & 3 & 1 & 7 & 1 & 0 \\
\hline & $\begin{array}{c}\text { El Kawady } \\
(\mathrm{k})\end{array}$ & 3 & 2 & 1 & 10 & 10 & 0 & 3 & 1 & 2 & 6 & 0 & 1 \\
\hline $\begin{array}{c}\text { Beni Suef } \\
(\text { Bs })\end{array}$ & $\begin{array}{c}\text { Kafr Mymon } \\
\text { (y) }\end{array}$ & 0 & 0 & 0 & 9 & 9 & 0 & 2 & 1 & 1 & 4 & 0 & 0 \\
\hline $\operatorname{Giza}(\mathbf{G})$ & Wardan (d) & 0 & 0 & 0 & 36 & 31 & 0 & 3 & 2 & 1 & 3 & 1 & 0 \\
\hline \multicolumn{2}{|c|}{ Total } & 21 & 18 & 3 & 160 & 147 & $\mathbf{0}$ & 36 & 16 & 8 & 61 & 6 & 1 \\
\hline
\end{tabular}

TSI= Total suspected isolates, $(\mathrm{t})=$ Typical $R$. solanacearum form, (a) = Atypical $R$. solanacearum form.

Table 5. Preliminary identification of Ralstonia solanacearum isolates of the cultivated common field crops in some Egyptian governorates using SMSA test

\begin{tabular}{|c|c|c|c|c|c|c|c|}
\hline \multirow{3}{*}{ Governorate } & \multirow{3}{*}{ Location } & \multicolumn{6}{|c|}{$\begin{array}{l}\text { No. of typical and atypical isolates from other } \\
\text { common field crops }\end{array}$} \\
\hline & & \multicolumn{3}{|c|}{ Plant } & \multicolumn{3}{|c|}{ Soil } \\
\hline & & TSI & $\mathbf{t}$ & $\bar{A}$ & TSI & $\mathbf{T}$ & $\mathbf{a}$ \\
\hline \multirow{4}{*}{$\begin{array}{c}\text { Gharbia } \\
\text { (Gh) }\end{array}$} & & $2 \mathrm{~T}$ & & & $3 \mathrm{~T}$ & & \\
\hline & Kafr EL zayat (z) & $\mathrm{OE}$ & 0 & 0 & $6 \mathrm{E}$ & $2 \mathrm{E}$ & $1 \mathrm{~T}$ \\
\hline & & 10 & & & 20 & & \\
\hline & Gafarya (g) & $\begin{array}{l}0 \mathrm{C} \\
0 \mathrm{Cl}\end{array}$ & 0 & 0 & $\begin{array}{l}1 \mathrm{C} \\
2 \mathrm{Cl}\end{array}$ & 0 & 0 \\
\hline Beheira (Bh) & Kom Hamada (h) & $1 \mathrm{~T}$ & 0 & 0 & $2 \mathrm{~T}$ & 0 & $1 \mathrm{~T}$ \\
\hline \multirow{6}{*}{ Menofia (M) } & Talia (i) & $\begin{array}{c}0 \mathrm{Ca} \\
0 \mathrm{Sb}\end{array}$ & 0 & 0 & $\begin{array}{l}1 \mathrm{Ca} \\
1 \mathrm{Sb}\end{array}$ & 0 & 0 \\
\hline & & OB & & & $2 \mathrm{~B}$ & & \\
\hline & $\Omega m$ Saher $(m)$ & $\mathrm{OSb}$ & 0 & 0 & $1 \mathrm{Sb}$ & $1 \mathrm{~B}$ & \\
\hline & Um saber (m) & $0 \mathrm{C}$ & 0 & 0 & $1 \mathrm{C}$ & $1 \mathrm{Sb}$ & 0 \\
\hline & & $0 \mathrm{Cl}$ & & & $2 \mathrm{Cl}$ & & \\
\hline & El Kawady (k) & $1 \mathrm{P}$ & 0 & 0 & $1 \mathrm{P}$ & 0 & $1 \mathrm{P}$ \\
\hline \multirow{2}{*}{$\begin{array}{l}\text { Beni Suef } \\
\text { (Bs) }\end{array}$} & Kafr Mymon (y) & $0 \mathrm{O}$ & 0 & 0 & $2 \mathrm{O}$ & $1 \mathrm{~T}$ & 0 \\
\hline & & $1 \mathrm{~T}$ & 0 & 0 & $1 \mathrm{~T}$ & TT & 0 \\
\hline \multirow{2}{*}{ Giza (G) } & Wardan (d) & $1 \mathrm{P}$ & 0 & 0 & $3 \mathrm{P}$ & $1 \mathrm{P}$ & 0 \\
\hline & & $1 \mathrm{~T}$ & 0 & 0 & $4 \mathrm{~T}$ & $1 \mathrm{~T}$ & 0 \\
\hline \multicolumn{2}{|c|}{ Total } & 8 & $\overline{0}$ & $\mathbf{0}$ & 35 & 7 & 3 \\
\hline
\end{tabular}

Where: $\mathrm{P}=$ Pepper, $\mathrm{T}=$ Tomato, $\mathrm{B}=$ Banana, $\mathrm{Sb}=$ Snap Beans, $\mathrm{E}=$ Eggplant, $\mathrm{t}=$ Typical $R$. solanacearum form, a=Atypical $R$. solanacearum form. 
Table 6. Total number of Ralstonia solanacearum isolates, which identified using IFAS test and SMSA medium

\begin{tabular}{|c|c|c|c|c|c|c|c|}
\hline \multirow[t]{2}{*}{ Governorate } & \multirow[t]{2}{*}{ Source } & \multirow[t]{2}{*}{ Habitats } & \multirow[t]{2}{*}{ Total samples } & \multicolumn{2}{|c|}{$\begin{array}{c}\text { Positive sample } \\
\text { IF } \\
\end{array}$} & \multicolumn{2}{|c|}{$\begin{array}{c}\text { Positive typical } \\
\text { SMSA } \\
\end{array}$} \\
\hline & & & & no. & $\%$ & no. & $\%$ \\
\hline \multirow{6}{*}{ Gharbia (Gh) } & \multirow{4}{*}{$\begin{array}{l}\text { Potato } \\
\text { field }\end{array}$} & Water & 5 & 5 & 100.0 & 4 & 80.0 \\
\hline & & Tuber & 68 & 63 & 92.6 & 63 & 86.7 \\
\hline & & Soil & 16 & 4 & 25.0 & 3 & 18.7 \\
\hline & & Weed & 29 & 3 & 10.3 & 3 & 10.3 \\
\hline & \multirow{2}{*}{$\begin{array}{l}\text { Other } \\
\text { crops }\end{array}$} & Plant & 3 & 0 & 0.0 & 0 & 0.0 \\
\hline & & Soil & 14 & 3 & 21.4 & 2 & 14.2 \\
\hline \multirow{6}{*}{ Beheira (Bh) } & \multirow{4}{*}{$\begin{array}{l}\text { Potato } \\
\text { field }\end{array}$} & Water & 9 & 9 & 100.0 & 8 & 88.8 \\
\hline & & Tuber & 7 & 6 & 85.7 & 6 & 85.7 \\
\hline & & Soil & 6 & 6 & 100.0 & 4 & 66.6 \\
\hline & & Weed & 7 & 0 & 0.0 & 0 & 0.0 \\
\hline & \multirow{2}{*}{$\begin{array}{l}\text { Other } \\
\text { crops }\end{array}$} & Plant & 1 & 0 & 0.0 & 0 & 0.0 \\
\hline & & Soil & 2 & 1 & 50.0 & 0 & 0.0 \\
\hline \multirow{6}{*}{$\begin{array}{l}\text { Menofia } \\
\text { (M) }\end{array}$} & \multirow{4}{*}{$\begin{array}{l}\text { Potato } \\
\text { field }\end{array}$} & Water & 7 & 7 & 100.0 & 6 & 85.7 \\
\hline & & Tuber & 40 & 38 & 95.0 & 38 & 95.0 \\
\hline & & Soil & 9 & 9 & 100.0 & 6 & 66.6 \\
\hline & & Weed & 18 & 3 & 16.6 & 2 & 11.1 \\
\hline & \multirow{2}{*}{$\begin{array}{l}\text { Other } \\
\text { crops }\end{array}$} & Plant & 1 & 0 & 0.0 & 0 & 0.0 \\
\hline & & Soil & 9 & 3 & 33.3 & 2 & 18.1 \\
\hline \multirow{6}{*}{$\begin{array}{l}\text { Beni Suef } \\
\quad(\text { Bs })\end{array}$} & \multirow{4}{*}{$\begin{array}{l}\text { Potato } \\
\text { field }\end{array}$} & Water & 0 & 0 & 0.0 & 0 & 0.0 \\
\hline & & Tuber & 9 & 9 & 100.0 & 9 & 100.0 \\
\hline & & Soil & 2 & 2 & 100.0 & 1 & 50.0 \\
\hline & & Weed & 4 & 0 & 0.0 & 0 & 0.0 \\
\hline & \multirow{2}{*}{$\begin{array}{l}\text { Other } \\
\text { crops }\end{array}$} & Plant & 1 & 0 & 0.0 & 0 & 0.0 \\
\hline & & Soil & 3 & 1 & 33.3 & 1 & 33.3 \\
\hline \multirow[t]{7}{*}{ Giza (G) } & \multirow{4}{*}{$\begin{array}{l}\text { Potato } \\
\text { field }\end{array}$} & Water & 0 & 0 & 0.0 & 0 & 0.0 \\
\hline & & Tuber & 36 & 31 & 86.1 & 31 & 86.1 \\
\hline & & Soil & 3 & 3 & 100.0 & 2 & 66.6 \\
\hline & & Weed & 3 & 1 & 33.3 & 1 & 33.3 \\
\hline & \multirow{2}{*}{$\begin{array}{l}\text { Other } \\
\text { crops }\end{array}$} & Plant & 2 & 0 & 0.0 & 0 & 0.0 \\
\hline & & Soil & 7 & 2 & 28.2 & 2 & 28.2 \\
\hline & Total & & 321 & 209 & & 194 & \\
\hline
\end{tabular}

Table 7. Selected Ralstonia solanacearum isolates for further tests

\section{Governorate}

Typical isolates $(\mathbf{t})$

Atypical Isolates (a)

\begin{tabular}{ccccccccccc} 
& Wa & T & S & We & O & Wa & T & S & We & O \\
\hline Gharbia $(\mathbf{G h})$ & 2 & 5 & 2 & 3 & 2 & 1 & 0 & 1 & 0 & 1 \\
\hline Beheira $(\mathbf{B h})$ & 2 & 2 & 3 & 0 & 0 & 1 & 0 & 2 & 0 & 1 \\
\hline Menofia $(\mathbf{M})$ & 3 & 3 & 3 & 2 & 2 & 1 & 0 & 3 & 1 & 1 \\
\hline Beni Suef $(\mathbf{B s})$ & 0 & 4 & 1 & 0 & 1 & 0 & 0 & 1 & 0 & 0 \\
\hline Giza $(\mathbf{G})$ & 0 & 6 & 2 & 1 & 2 & 0 & 0 & 1 & 0 & 0 \\
\hline Sub-Total & $\mathbf{7}$ & $\mathbf{2 0}$ & $\mathbf{1 1}$ & $\mathbf{6}$ & $\mathbf{7}$ & $\mathbf{3}$ & $\mathbf{0}$ & $\mathbf{8}$ & $\mathbf{1}$ & $\mathbf{3}$ \\
\hline Total & & & & & & &
\end{tabular}

Where: $\mathrm{Wa}=$ Water isolates, $\mathrm{T}=$ Tuber isolates, $\mathrm{S}=$ Soil isolates, $\mathrm{We}=\mathrm{Weed}$ isolates and $\mathrm{O}=$ other common crops

Table 8. Code of selected sixty-six isolates of Ralstonia solanacearum isolates

\begin{tabular}{llllllll}
\hline No. & Code & No. & Code & No. & Code & No. & Code \\
\hline 1 & GhztWa & 18 & BsytT & 35 & MktS & 52 & GhzaWa \\
\hline 2 & GhgtWa & 19 & BsytT & 36 & BsytS & 53 & BhhaWa \\
\hline 3 & BhhtWa & 20 & BsytT & 37 & GdtS & 54 & MkaWa \\
\hline
\end{tabular}




\begin{tabular}{llllllll}
\hline 4 & BhhtWa & 21 & BsytT & 38 & GdtS & 55 & GhzaS \\
\hline 5 & MitWa & 22 & GdtT & 39 & GhztWe & 56 & BhhaS \\
\hline 6 & MmtWa & 23 & GdtT & 40 & GhgtWe & 57 & BhhaS \\
\hline 7 & MktWa & 24 & GdtT & 41 & GhgtWe & 58 & MmaS \\
\hline 8 & GhztT & 25 & GdtT & 42 & MitWe & 59 & MkaS \\
\hline 9 & GhztT & 26 & GdtT & 43 & MmtWe & 60 & MkaS \\
\hline 10 & GhztT & 27 & GdtT & 44 & GdtWe & 61 & BsyaS \\
\hline 11 & GhgtT & 28 & GhztS & 45 & GhztOE & 62 & GdaS \\
\hline 12 & GhgtT & 29 & GhgtS & 46 & GhztOE & 63 & MkaWe \\
\hline 13 & BhhtT & 30 & BhhtS & 47 & MmtOB & 64 & GhzaOT \\
\hline 15 & BhhtT & 31 & BhhtS & 48 & MmtOSb & 65 & BhhaOT \\
\hline 16 & MitT & 32 & BhhtS & 49 & BsytOT & 66 & MkaOP \\
\hline 17 & MmtT & 33 & MitS & 50 & GdtOP & & \\
\hline WhtT & 34 & MmtS & 51 & GdtOT & & \\
\hline
\end{tabular}

Where: Gh= Gharbia, Bh= Beheira, M= Menofia, Bs= Beni Swef, G= Giza, z= Kafr el zayat, g= Gafarya, i= Talia, $\mathrm{m}=$ Om saber, $\mathrm{k}=$ Kawady, $\mathrm{h}=$ Kom Hamada, $\mathrm{y}=$ Kafr Mymon, $\mathrm{d}=$ Wardan, $\mathrm{t}=$ typical form, $\mathrm{a}=$ atypical form, $\mathrm{Wa}=$ water, $\mathrm{T}=$ Tuber, $\mathrm{S}=\mathrm{Soil}, \mathrm{We}=\mathrm{Weed}, \mathrm{O}=$ other crop soil, $\mathrm{E}=$ Eggplant, $\mathrm{B}=\mathrm{Banana}, \mathrm{Sb}=\mathrm{Snab} \mathrm{Beans}, \mathrm{T}=$ Tomato, $\mathrm{P}=$ Pepper

\section{Virulence of selected sixty-six isolates of $R$. solanacearum:}

Data in Tables $(9 \mathbf{9} \& 9 b)$ indicate that all tested 66 isolates of typical or atypical $R$. solanacearum could infect potato plants (cv. Spunta) where they caused disease severity \% (DS \%) ranged between $26.4 \%-100 \%$ when incubated for 20 days. In this respect, MktT-17 (Menofia - EL Kawady - typical tuber) isolate was the highest infective one where it caused 100\% DS followed by MmtT-16 (MenofiaOm Saber -typical -tuber) and GhztT-8 (Gharbia Kafr el Zayat - typical - tuber) which caused $98.4 \%$ DS at 20 days of incubation period. On the other hand, the least infective isolates were BhhaWa-53 (Beheira -Kom Hamada - atypical - water), BhhaS-56 (Beheira- Kom Hamada - atypical - soil) and GdaS-62 (Giza -Wardan - atypical - soil) at the same period of incubation. Also, the rest of other isolates were infective and caused DS\% of bacterial wilt symptoms below $98.4 \%$ and over $28.0 \%$. It is clear from the obtained results that all atypical forms of $R$. solanacearum isolates were less infective than the typical forms which were more virulent in this respect. On the other hand, all sixty-six tested isolates of typical or atypical $R$. solanacearum were also infective and caused bacterial wilt symptoms of tomato plants (cv. Ponto). In this respect, all tested isolates caused $100 \%$ DS of wilt infection but they differed in the time of incubation that taken. Also, the typical isolates were more virulent and fast in their infection on tomato plants (cv. Ponto) where atypical forms took a long time of incubation till the DS reached $100 \%$. The highest DS\% on tomato plants (cv. Ponto) was recorded with the typical $R$. solanacearum isolate MktT-17 (Menofia - EL Kawady - typical tuber) to be $100 \%$ at 4 days of incubation period. While, the lowest DS\% was recorded with MmtOB47 (Menofia - Om saber - typical - other crop Banana) where it recorded $100 \%$ DS at 10 days of incubation period. Moreover, the fastest atypical form of $R$. solanacearum was GhzaS-55 (Gharbia - Kafr el Zayat - atypical - Soil) which causing $100 \%$ wilt DS on tomato plants (cv. Ponto) at 9 days of incubation period while, the slowest atypical ones were BhhaS56 (Beheira - Kom Hamada - atypical - soil), GdaS62 (Giza - Wardan - atypical - soil) and GHzaOT-64 (Gharbia- Kafr el zayat atypical- other crop - Tomato) which caused $100 \%$ wilt DS on tomato plants (cv. Ponto) at 15 days of incubation period. These results are in harmony with those obtained by Aley $\boldsymbol{e t} \boldsymbol{a l}$. (1994) who inoculated the purified culture of $R$. solanacearum on King's medium on tomato or potato seedlings to confirm its pathogenicity. Wilting of tomato seedlings may begin in less than a week, but potato seedling will appear within 4 weeks. Also, Elphinstone et al. (1996) reported that the bioassay test using tomato seedlings is reliable to detect as few as $10^{4}$ cells per $\mathrm{ml}$ of suspension of infected potato extract. Tomato seedlings are widely used for both pathogenicity testing. Depending on the inoculum potential of the bacterium and the availability of optimum environmental conditions, typical wilting symptoms are usually apparent within a week of inoculation. However, El-Ariqi et al. (2005) showed that isolates of $R$. solanacearum displayed varying levels of virulence on potato Spunta and Diamont cultivars. Mikhail et al. (2012) stated that all the nine isolates of $R$. solanacearum (Six virulent and three avirulent) recovered from the natural habitats (potato tubers, weeds, soil and water) were pathogenic to potato plants causing different symptoms. Also, ElHaj Saleh (2014) found that when potato plants were injected with $R$. solanacearum isolates, wilt symptoms appeared compared to non-injected potato plants this results confirms that all the tested isolates are virulent and belong to race 3 biovar 2. Hagag (2015) found that number of positive $R$. solanacearum isolates retrieved from infected tubers was higher than isolates retrieved from soil followed by weed isolates also, positive samples were highly recorded in Beheria 
governorate followed by Menofia governorate and the lowest recorded was in Ben-Seweif governorate. Moreover, Mikhail et al. (2017) found that all tested virulent isolates of $R$. solanacearum showed high percentages of infection and disease severity on tomato plants after 5 days while, avirulent isolates showed low percentages of infection and disease severity on tomato plants causing stunting and chlorosis.

Table 9a. Virulence of sixty-six isolates of identified $R$. solanacearum on potato plants (cv. Spunta) under artificial inoculation conditions for 20 days.

\begin{tabular}{|c|c|c|c|c|c|c|c|c|}
\hline No. & $\begin{array}{c}\text { Tested } \\
\text { isolates }\end{array}$ & DS\% & No. & Tested isolates & DS\% & No. & $\begin{array}{c}\text { Tested } \\
\text { isolates }\end{array}$ & DS\% \\
\hline 1 & GhztWa & 79.2 & 23 & GdtT & 76.0 & 45 & GhztOE & 52.8 \\
\hline 2 & GhgtWa & 64.8 & 24 & GdtT & 82.4 & 46 & GhztOE & 59.2 \\
\hline 3 & BhhtWa & 59.2 & 25 & GdtT & 68.8 & 47 & $\mathrm{MmtOB}$ & 48.8 \\
\hline 4 & BhhtWa & 63.2 & 26 & GdtT & 63.2 & 48 & $\mathrm{MmOSb}$ & 54.4 \\
\hline 5 & MitWa & 80.8 & 27 & GdtT & 88.0 & 49 & BsytOT & 63.2 \\
\hline 6 & $\mathrm{MmtWa}$ & 85.6 & 28 & GhztS & 72.0 & 50 & GdtOP & 68.8 \\
\hline 7 & MktWa & 87.2 & 29 & GhgtS & 68.8 & 51 & GdtOT & 59.2 \\
\hline 8 & GhztT & 98.4 & 30 & BhhtS & 72.0 & 52 & GhzaWa & 30.4 \\
\hline 9 & GhztT & 87.2 & 31 & BhhtS & 76.0 & 53 & BhhaWa & 26.4 \\
\hline 10 & GhztT & 91.2 & 32 & BhhtS & 76.0 & 54 & MkaWa & 34.4 \\
\hline 11 & GhgtT & 85.2 & 33 & MitS & 85.6 & 55 & GhzaS & 48.0 \\
\hline 12 & GhgtT & 87.2 & 34 & $\mathrm{MmtS}$ & 88.0 & 56 & BhhaS & 27.2 \\
\hline 13 & BhhtT & 88.0 & 35 & $\mathrm{MktS}$ & 92.0 & 57 & BhhaS & 30.4 \\
\hline 14 & BhhtT & 87.2 & 36 & GdtS & 79.2 & 58 & MmaS & 30.4 \\
\hline 15 & MitT & 96.8 & 37 & GdtS & 72.0 & 59 & $\mathrm{MkaS}$ & 54.4 \\
\hline 16 & MmtT & 98.4 & 38 & GhztWe & 68.8 & 60 & MkaS & 48.8 \\
\hline 17 & MktT & 100.0 & 39 & GhgtWe & 80.8 & 61 & Bsyas & 36.4 \\
\hline 18 & BsytT & 88.0 & 40 & GhgtWe & 80.8 & 62 & GdaS & 28.0 \\
\hline 19 & BsytT & 87.2 & 41 & MitWe & 72.0 & 63 & MkaWe & 48.2 \\
\hline 20 & BsytT & 82.4 & 42 & MmtWe & 76.0 & 64 & GhzaOT & 40.8 \\
\hline 21 & BsytT & 85.6 & 43 & GdtWe & 82.4 & 65 & BhhaOT & 54.4 \\
\hline 22 & GdtT & 76.0 & 44 & GhztOE & 68.2 & 66 & $\mathrm{MkaOP}$ & 40.8 \\
\hline
\end{tabular}

Where: DS \%= Disease Severity\%, Gh= Gharbia, Bh= Beheira, M= Menofia, Bs= Beni Suef, G= Giza, Z= Kafr el zayat, $\mathrm{g}=$ Gafarya, $\mathrm{i}=$ Talia, $\mathrm{m}=$ Om saber, $\mathrm{k}=$ Kawady, $\mathrm{h}=$ Kom Hamada, $\mathrm{y}=$ Kafr Mymon, $\mathrm{d}=\mathrm{Wardan}, \mathrm{t}=$ typical form, $\mathrm{a}=$ atypical form, $\mathrm{Wa}=$ water, $\mathrm{T}=$ Tuber, $\mathrm{S}=$ Soil, $\mathrm{We}=\mathrm{Weed}, \mathrm{O}=$ other crop soil, $\mathrm{E}=\mathrm{Eggplant}$, $\mathrm{B}=$ Banana, $\mathrm{Sb}=$ Snap Beans, $\mathrm{T}=$ Tomato, $\mathrm{P}=$ Pepper

Table 9b. Virulence of sixty-six isolates of identified $R$. solanacearum on Tomato plants (cv. Ponto) under artificial inoculation conditions.

\begin{tabular}{cccccccccccc}
\hline No. & $\begin{array}{c}\text { Tested } \\
\text { isolates }\end{array}$ & $\begin{array}{c}\text { IP } \\
\text { (days) }\end{array}$ & $\begin{array}{c}\text { DS } \\
\mathbf{\%}\end{array}$ & $\mathbf{N o .}$ & $\begin{array}{c}\text { Tested } \\
\text { isolates }\end{array}$ & $\begin{array}{c}\text { IP } \\
\text { (days) }\end{array}$ & $\begin{array}{c}\text { DS } \\
\boldsymbol{\%}\end{array}$ & No. & $\begin{array}{c}\text { Tested } \\
\text { isolates }\end{array}$ & $\begin{array}{c}\text { IP } \\
\text { (days) }\end{array}$ & $\begin{array}{c}\text { DS } \\
\boldsymbol{\%}\end{array}$ \\
\hline 1 & GhztWa & 7 & 100 & 23 & GdtT & 8 & 100 & 45 & GhztOE & 9 & 100 \\
\hline 2 & GhgtWa & 8 & 100 & 24 & GdtT & 7 & 100 & 46 & GhztOE & 9 & 100 \\
\hline 3 & BhhtWa & 8 & 100 & 25 & GdtT & 8 & 100 & 47 & MmtOB & 10 & 100 \\
\hline 4 & BhhtWa & 7 & 100 & 26 & GdtT & 8 & 100 & 48 & MmOSb & 9 & 100 \\
\hline 5 & MitWa & 8 & 100 & 27 & GdtT & 6 & 100 & 49 & BstOT & 8 & 100 \\
\hline 6 & MmtWa & 7 & 100 & 28 & GhztS & 8 & 100 & 50 & GdtOP & 8 & 100 \\
\hline 7 & MktWa & 7 & 100 & 29 & GhgtS & 9 & 100 & 51 & GdtOT & 9 & 100 \\
\hline 8 & GhztT & 8 & 100 & 30 & BhhtS & 7 & 100 & 52 & GhzaWa & 10 & 100 \\
\hline 9 & GhztT & 6 & 100 & 31 & BhhtS & 8 & 100 & 53 & BhhaWa & 14 & 100 \\
\hline 10 & GhztT & 5 & 100 & 32 & BhhtS & 7 & 100 & 54 & MkaWa & 12 & 100 \\
\hline 11 & GhgtT & 7 & 100 & 33 & MitS & 6 & 100 & 55 & GhzaS & 9 & 100 \\
\hline 12 & GhgtT & 6 & 100 & 34 & MmtS & 6 & 100 & 56 & BhhaS & 15 & 100 \\
\hline 13 & BhhtT & 7 & 100 & 35 & MktS & 6 & 100 & 57 & BhhaS & 14 & 100 \\
\hline 14 & BhhtT & 6 & 100 & 36 & GdtS & 8 & 100 & 58 & MmaS & 12 & 100 \\
\hline 15 & MitT & 6 & 100 & 37 & GdtS & 8 & 100 & 59 & MkaS & 12 & 100 \\
\hline
\end{tabular}




\begin{tabular}{cccccccccccc}
\hline 16 & MmtT & 4 & 100 & 38 & GhztWe & 9 & 100 & 60 & MkaS & 12 & 100 \\
\hline 17 & MktT & 4 & 100 & 39 & GhgtWe & 8 & 100 & 61 & BsyaS & 14 & 100 \\
\hline 18 & BsytT & 5 & 100 & 40 & GhgtWe & 8 & 100 & 62 & GdaS & 15 & 100 \\
\hline 19 & BsytT & 6 & 100 & 41 & MitWe & 7 & 100 & 63 & MkaWe & 14 & 100 \\
\hline 20 & BsytT & 6 & 100 & 42 & MmtWe & 8 & 100 & 64 & GhzaOT & 15 & 100 \\
\hline 21 & BsytT & 6 & 100 & 43 & GdtWe & 8 & 100 & 65 & BhhaOT & 12 & 100 \\
\hline 22 & GdtT & 8 & 100 & 44 & GhztOE & 8 & 100 & 66 & MkaOP & 13 & 100 \\
\hline
\end{tabular}

Where: $\mathrm{DS} \%=$ Disease Severity\%, IP= Incubation period (days)Gh= Gharbia, Bh= Beheira, M= Menofia, Bs= Beni Suef, G= Giza, Z= Kafr el zayat, $\mathrm{g}=$ Gafarya, $\mathrm{i}=$ Talia, $\mathrm{m}=$ Om saber, $\mathrm{k}=$ Kawady, $\mathrm{t}=$ typical form, $\mathrm{a}=$ atypical form, $\mathrm{Wa}=$ water, $\mathrm{T}=$ Tuber, $\mathrm{S}=\mathrm{Soil}$, $\mathrm{We}=\mathrm{Weed}, \mathrm{O}=$ other crop soil, $\mathrm{E}=$ Eggplant, $\mathrm{B}=\mathrm{B}$ anana, $\mathrm{Sb}=\mathrm{Snap} \mathrm{B}$ eans, $\mathrm{T}=$ Tomato, $\mathrm{P}=$ Pepper

\section{References}

Abd El-Ghany, H. (2010). Ecological and molecular studies on Ralstonia solanacearum and its control in Egypt. PhD. Theses. Institute of Environmental Studies \& Research, Ain Shams University.

Aley, P.; French, E. and Nydegger, U. (1994). Methods of greenhouse inoculation with Pseudomonas solanacearum to select resistant potato clones. Fitopatologia 20:29 (Abstract).

Ali, E.E. (2017). Sensitivity of different detection methods of Ralstonia solanacearum in potato tubers and infected sources. M.Sc. Theses. Fac. of Agri., Cairo University.

Atta, K.M. (2008). Studying the interaction between potato brown rot bacterium and root exudates in certain crops. Msc. Theses. Fac. of science, Benha University.

Dowson, W.J. (1957). Plant disease due to bacteria. Second edition. Cambridge at the University Press, PP. 232.

El-Ariqi, S.N.S.; El-Mflehi, M.; El-Abara, K.; ElKobati, A., and Shaari, A. (2005). Antibacterial activity of extracts from Withania somnifera and Aloe vera against Ralstonia solanacearum in potato. Arab Journal of Plant Protection, 23:95-99.

El- Haj Saleh, O.M.E (2014). Studies on some microorganisms as biological control agents for potato brown rot. M.Sc. Theses .Fac. of Agri., Cairo University. Elphinstone, J.G. (1996). Survival and Possibilities for extinction of Pseudomonas solanacearum (Smith) in cool climates. Potato Res., 39: 403410.

Elphinstone, J.G.; Hennessy, J.; Wilson, J.K. and Stead, D.E. (1996): Sensitivity of different methods for the detection of Ralstonia solanacearum in potato tuber extracts. Bulletin OEEP/EPPO Bulletin, 26: 663-678.

Engelbrecht, M.C. (1994). Modification of a semiselective medium for the isolation and quantification of Pseudomonas solanacearum. ACIAR Bacterial Wilt Newsletter, 10: 3-5.

Fahy, P.C. and Persley, G.J. (1983). Plant bacterial disease. A Diagnostic Guide. Academic Press, New York.
FAO STAT (2013). http://www.fao.org/faostat FAO STAT (2016). http://www.fao.org/faostat Farag, N.S. (2000). Spotlights on potato brown rot in Egypt. Proc. $9^{\text {th }}$ Congress of the Egypt. Phytopathol. Soc., May, 405-408.

Fegan M \& Prior P (2005): How complex is the Ralstonia solanacearum species complex. Bacterial wilt disease and the Ralstonia solanacearum species complex, (Allen C, Prior P \& Hayward AC, eds.), pp. 449-461. APS Press, Saint Paul, USA.

Hagag, M.H.E.M. (2015): Use of Soil Amendments to Control Bacterial Wilt Disease in Potato. M.Sc. Thesis, Plant Pathology Department, Faculty of Agriculture, Ain Shams University.

Hayward, A.C. (1991). Biology and epidemiology of bacterial wilt caused by Pseudomonas solanacearum. Annual Review of phytopathology 29: 65-87.

Janse, J. D. (1996): Potato brown rot in Western Europe - history, present occurrence and some remarks on possible origin, epidemiology and control strategies. OEPP/ EPPO Bulletin 26: $679-695$.

Janse, J. D. and Schans, J. (1998). Experiences with the diagnosis and epidemiology of bacterial brown rot ( $R$. solanacearum) in the Netherlands. Bulletin OEPP/EPPO Bulletin, 28: 65-67.

Janse, J.D. (1988). A detection methods for Pseudomonas solanacearum in symptomless potato tubers and some data on its sensitivity and specificity. EPPO Bulletin, 18: 343-351.

Kelman, A. (1954). The relationship of pathogenicity in Pseudomonas solanacearum to colony appearance on tetrazolium medium. Phytopathology, 44: 693- 695.

Kempe, J. and Sequeira L. (1983). Biological control of bacterial wilt of Potatoes: Attempts to induce resistance by treating tubers with bacteria. Plant Dis. 67:499-503.

Martin, J.E. and El-Nashaar, H.M. (1992). Pathogenicity of the new phenotypes of Pseudomonas solanacearum from peru. ACIAR Proceeding 45: 78- 84.

Mikhail, M.S.; Mohamed, M.E.; Abdel-Alim, A.I. and Youssef, M.M. (2012). Using virulence genes $h r p B, e g l$ and $f l i C$ in differentiation 
between virulent and avirulent isolates of Ralstonia solanacearum, the causal agent of potato brown rot. African Journal of Microbiology Research, 6(2): 225-235.

Mikhail, M.S.; Abdel-Alim, A.I.; Balabel. N.M. and Abbas, A.M. (2017). Virulence of Ralstonia solanacearum phylotype ii sequevar $i$, the causal pathogen of potato brown rot. $j$. biol. chem. environ. sci., vol. 21(3): 219-235

Pradhanang, P. M.; Elphinstone, J. G. and Fox, R. T. V. (2000). Identification of crop and weed hosts of $R$. solanacearum biovar 2 in the hills of Nepal. Plant Pathology, 49: 403 - 413.

Smith, E.F. (1896). A bacterial disease of the tomato, eggplant and Irish potato. U. S. Dept. Agric. Div. Veg. Phys. Path. Bull., 12: 1-26.

Smith, E.F. (1914). Bacteria in relation to plant disease. Carnegie Inst. Wash., 3: 178.

Wenneker, M.; Verdel, M. S. W.; Groeneveld, R. M. W.; Kempenaar, C.; van Beuningen, A. R. and Janse, J. D. (1999). Ralstonia (Pseudomonas) solanacearum race 3 (biovar 2 ) in surface water and natural weed hosts: first report on stinging nettle (Urtica dioica).
European Journal of Plant Pathology, 105: 307 -315 .

Yabuuchi, E.; Y. Kosako; H. Oyaizu; I. Yano; H. Hartta; Y. Hashimoto; T. Ezaki and $M$. Arakawa (1992). Proposal of Burkholderia gen. Nov. and transfer of seven species of the genus Pseudomonas group II to the new genus with the type species Burkholderia cepacia (Palleroni and Holmes 1981) Comb. Nov. Microbiology and Immunology, 36: 12511275.

Yabuuchi, E.; Kosako, Y.; Yano, I.; Hotta, H. and Nishiuchi, Y. (1995). Transfer of two Burkholderia and on Alcaligenes species to Ralstonia Gen. Nov. Proposal of Ralstonia picketii (Ralston, Palleroni and Doudoroff 1973) Comb. Nov. Ralstonia solanacearum (Smith, 1896) Comb. Nov. and Ralstonia eutropha (Davis, 1969) Comb. Nov. Microbiol. Immunol., 39(11): 897-904.

Zaki, M. A. (1991). Identification of important weeds of Egypt. Agricultural Production and Credit Project (APCP).

Zaki, M.A (2000). Identification and control of important weeds in Egypt. ARC Press. 


$$
\begin{aligned}
& \text { العصر والكشف السريع لبكتيريا رالستونيا سولانسيرم المسبب المرضي لمرض العفن البني علي البطاطس في بعض } \\
& \text { المحافظات المصرية } \\
& \text { محمد سليمان حنفي * ، جهاد محمد الهباء" ، ، فتحي جاد محمد *" ، نجلاء موسي بلابل " ، جمال عاشور أحمد *" }
\end{aligned}
$$

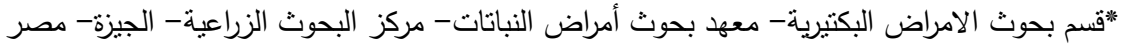

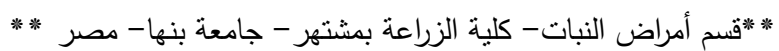

استخدت نباتات البطاطس المصابة طبيعيا والمأخوذة من بيئات مختلفة وتظهر عليها أعراض الذبول والعفن البني لعزل المسبب المرضي

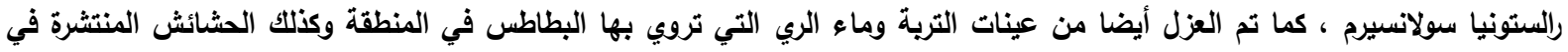

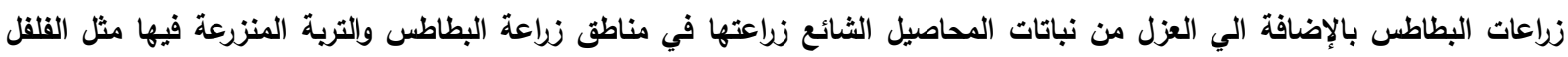

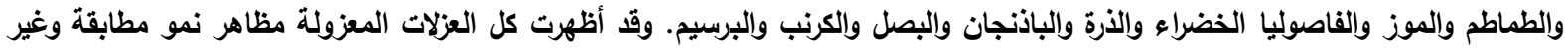

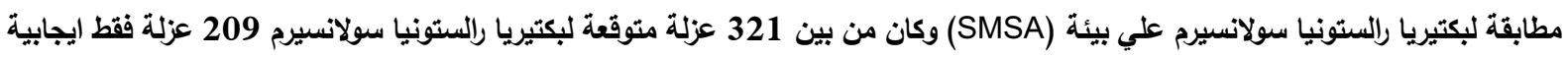

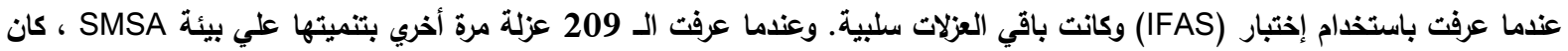

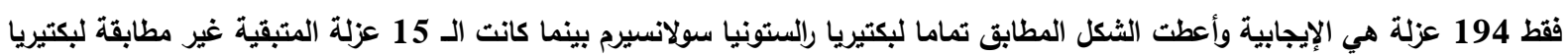

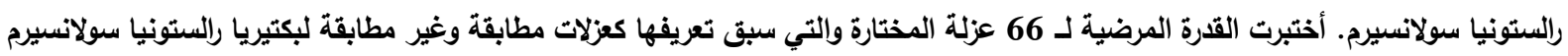

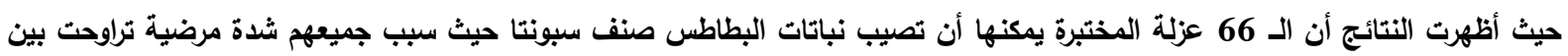

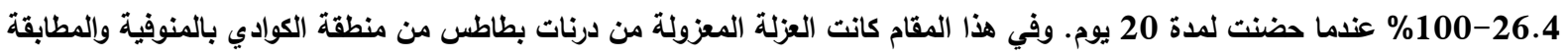
لبكتيريا رالستونيا سولانسيرم (MktT-17) هي أعلي العزلات إصابة للبطاطس حيث أعطت شدة إصابة 100\% بينما كانت أقل عزلة إصابة

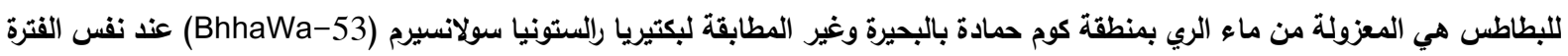

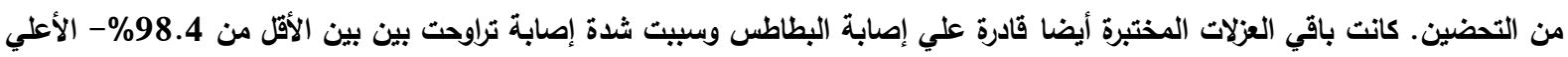

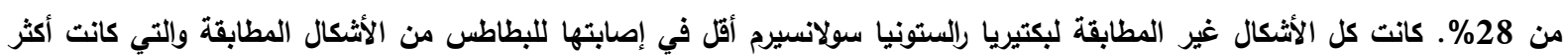

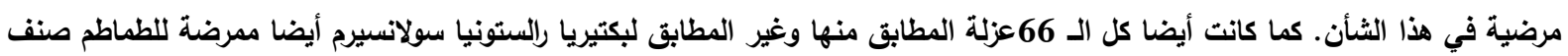

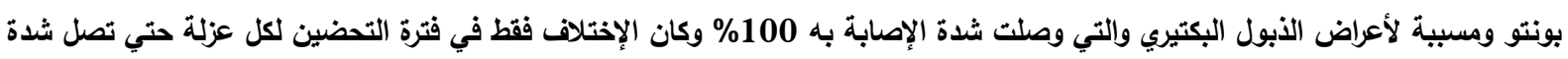

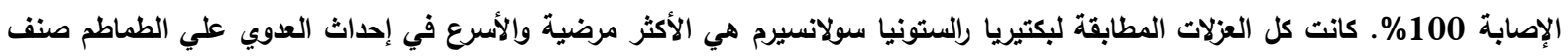

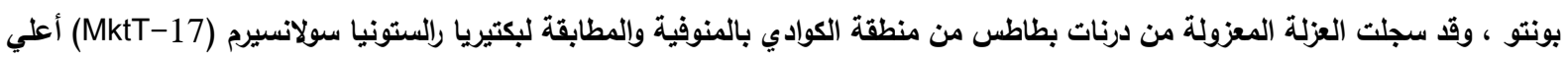

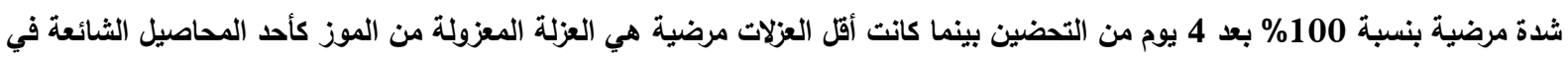

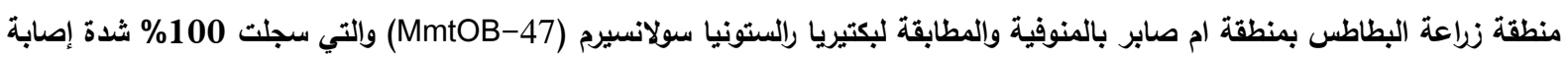

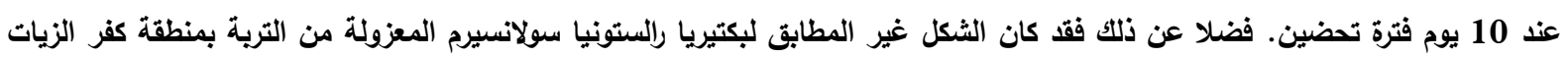

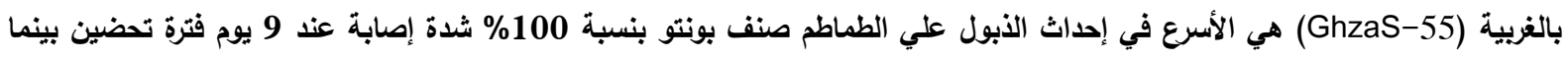

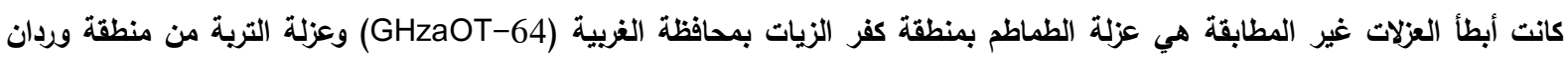

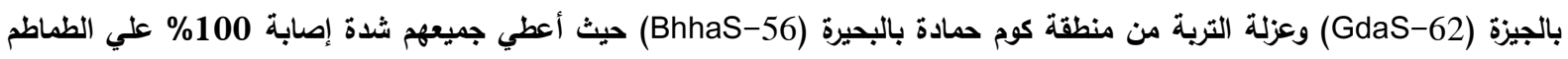
صنف بونتو عند 15 يوم فترة التحضين. 